Original research article

\title{
MLPA based detection of mutations in the dystrophin gene of 180 Polish families with Duchenne/Becker muscular dystrophy
}

\author{
Janusz G. Zimowski ${ }^{a, *}$, Diana Massalska ${ }^{c}$, Mariola Holding ${ }^{a}$, \\ Sylwia Jadczak ${ }^{a}$, Elżbieta Fidziańska ${ }^{a}$, Anna Łusakowska ${ }^{b}$, \\ Anna Kostera-Pruszczyk ${ }^{b}$, Anna Kamińska ${ }^{b}$, Jacek Zaremba ${ }^{a}$ \\ ${ }^{a}$ Department of Genetics, Institute of Psychiatry and Neurology, Warsaw, Poland \\ ${ }^{\mathrm{b}}$ Department of Neurology, Medical University of Warsaw, Warsaw, Poland \\ ${ }^{\mathrm{c}}$ I Department of Obstetrics and Gyneacology, Centre of Postgraduate Medical Education, Warsaw, Poland
}

\section{A R T I C L E I N F O}

\section{Article history:}

Received 23 April 2014

Accepted 15 October 2014

Available online 24 October 2014

Keywords:

Duchenne/Becker muscular

dystrophy

Molecular diagnostics

Multiplex ligation-dependent probe amplification

\begin{abstract}
A B S T R A C T
Duchenne/Becker muscular dystrophy (DMD/BMD) is a recessive, $\mathrm{X}$-linked disorder caused by a mutation in the dystrophin gene. Deletions account for approximately $60-65 \%$ of mutations, duplications for $5-10 \%$. The remaining cases are mainly point mutations. According to Monaco theory clinical form of the disease depends on maintaining or disrupting the reading frame. The purpose of the study was to determine frequency and location of deletions and duplications in the dystrophin gene, to determine the compliance between maintaining/disrupting the reading frame and clinical form of the disease and to check the effectiveness of MLPA (multiplex ligation-dependent probe amplification) in the detection of these mutations in hemizygous patients and heterozygous female carriers. The material is composed of combined results of molecular diagnosis carried out in years 20092012 in 180 unrelated patients referred with the diagnosis of DMD/BMD tested by use of MLPA. We identified 110 deletions, 22 duplication (in one patient two different duplications were detected) and 2 point mutations. Deletions involved mainly exons 45-54 and 3-21, whereas most duplications involved exons 3-18. The compliance with Monaco theory was $95 \%$ for deletions and $76 \%$ for duplications. Most of mutations in the dystrophin gene were localized in the hot spots - different for deletions and duplications. MLPA enabled their quick identification, exact localization and determination whether or not they maintained or disrupted the reading frame. MLPA was also effective in detection of deletions and duplications in female carriers.
\end{abstract}

(C) 2014 Polish Neurological Society. Published by Elsevier Urban \& Partner Sp. z o.o. All rights reserved.

\footnotetext{
* Corresponding author at: Department of Genetics, Institute of Psychiatry and Neurology, Al. Sobieskiego 9, 02-957 Warsaw, Poland. Tel.: +482245825 67; fax: +48228589169.

E-mail address: zimowski@ipin.edu.pl (J.G. Zimowski). http://dx.doi.org/10.1016/j.pjnns.2014.10.004

0028-3843/C 2014 Polish Neurological Society. Published by Elsevier Urban \& Partner Sp. z o.o. All rights reserved.
} 


\section{Introduction}

Duchenne/Becker muscular dystrophies (DMD/BMD) are genetic X-linked recessive disorders. DMD and BMD are the most common dystrophinopathies, with an incidence of 1:3500 and 1:1800 live birth males, respectively [1,2]. Both diseases lead to progressive irreversible muscle deterioration [3,4]. DMD shows an earlier onset and a more severe progression [5].

DMD and BMD are caused by mutations in the dystrophin gene $(2.4 \mathrm{Mb})$, localized on the chromosome Xp21.2 [6,7], comprises 79 exons encoding a $14 \mathrm{~kb}$ mRNA transcript [6]. The protein product, dystrophin, has a molecular weight of $427 \mathrm{kDa}$, connects extracellular matrix with cytoskeleton [8]. Dystrophin is missing in DMD patients $(<3 \%)$, whereas BMD patients have $10-40 \%$ of the normal protein or produce dystrophin of abnormal molecular weight [9].

Approximately $65 \%$ DMD cases and up to $85 \%$ of BMD cases are caused by large deletions in DMD gene $[6,10,11]$. Duplications account for approximately $5-10 \%$ of the mutations [12-15], whereas the remaining $25-30 \%$ are point mutations and microlesions [16].

According to Monaco's theory, frameshifting mutations generally cause more DMD, while in BMD the reading frame is usually intact. This frameshifting model complies with phenotype in $92 \%$. This rule, however, is more in keeping with deletions than with duplications in whom the reading of ORF is less predictable and that may be due to the fact that some duplications are inverted [17-20].

Most deletions cluster within two hot spot regions, the major site encompassing exons 44-52 (70\%) and a minor site including exons 3-19 (30\%). Duplications concentrate in proximal part of the dystrophin gene. Point mutations do not cluster in certain regions therefore they are more difficult to detect [16]. Since no effective therapy is available for DMD/BMD patients so far, the identification of female carriers is essential for genetic counseling and prenatal diagnosis. Although approximately $98 \%$ of deletions can be found in patients by using a multiplex polymerase chain reaction (PCR), this technique is not useful in detecting female carriers since deletions in one dystrophin allele are masked by the presence of the normal chromosomeX. Multiple ligation-dependent probe amplification (MLPA) is a new reliable method for detection deletions and duplications both in male patients and female carriers [21-23]. MLPA technique may also be a very useful tool for mutation detection in female carriers from DMD/BMD families, where DNA sample from a patient is not available.

\section{The aim of the studies}

1. Detection of deletions and duplications in 180 unrelated DMD/BMD patients by MLPA method.

2. Determination of size and frequency of the deletions and duplications found in the dystrophin gene of $180 \mathrm{DMD} / \mathrm{BMD}$ families.

3. Detection of deletions and duplications in 62 females, mothers of a single affected male, including 4 women from DMD families, in which DNA samples from probands were not available.
4. Verification of Monaco's theory for detected deletions and duplications.

\section{Materials}

A total of 180 DMD/BMD families entered this study. A group of 134 patients was diagnosed with DMD (Duchenne muscular dystrophy), 37 with BMD (Becker muscular dystrophy), 8 with DMD/BMD (presymptomatic period in very young patients) and 1 with IMD (intermediate form). In 4 DMD cases DNA from a patient was not available and DNA from the mothers was used for mutation analysis. Diagnosis was based on clinical presentation, family history, markedly elevated serum kinase levels and electromyography and progression of the disease, results immunohistochemistry in most of the cases was not available. In addition to these cases 62 mothers of a single affected male were added to this study, including 4 mothers of patients who deceased before molecular analysis was carried out. All the examined families originated from Poland. All the participants gave written informed consent to this study. The institute performing the study is a referral center receiving most samples from throughout the whole country.

\section{Methods}

DNA samples were obtained from peripheral blood lymphocytes according to standard procedures (MagNA Pure Compact, Roche). Mutations in the DMD gene were detected by multiple ligation-dependent probe amplification (MLPA) technique according to instructions of the manufacturer (MRC-Holland, Amsterdam, The Netherlands). Two sets of reagents for analysis of all 79 exons of the dystrophin gene were used: SALSA probe mix 034 (exons 1-10, 21-30, 41-50 and 61-70) and SALSA probe mix 035 (exons 11-20, 31-40, 51-60 and 71-79). Amplified products were run on ABI PRISM 3100 Genetic Analyzer. The obtained data were visually analyzed for the detection of deletions and duplications. Two or more peaks missing, corresponding to two or more exons, were representing a deletion. Absence of only one peak was additionally investigated by standard PCR method. If PCR did not confirm a deletion, amplified product of analyzed exon was subsequently sequenced according to Sanger [24].

\section{Results}

MLPA analysis showed the presence of 134 mutations in 133 DMD genes in 180 DMD/BMD patients (73.9\%). Deletions have been detected in 110 patients (61.1\%) (Table 1), duplications - in 21 (11.7\%) (Table 2), point mutations in - 2 (1.1\%) (c.134delA in exon $3, \mathrm{c} .5407 \mathrm{C}>\mathrm{T}$ in exon 38). Two independent duplications have been found in one family [25]. No mutations were identified in 47 probands (26.1\%). There were 75 DMD (75/134, 56.0\%), 29 BMD (29/37, 78.4\%), 5 DMD/BMD (5/8, 62.5\%) and $1 \mathrm{IMD}(1 / 1)$ patients with deletions and $17 \mathrm{DMD}(17 / 134,12.7 \%)$, $3 \mathrm{BMD}$ (3/37, 8.1\%), 1 DMD/BMD probands with duplications. Two DMD patients had point mutations. 
Table 1 - Deletions in DMD gene detected by use of MLPA in the group of 180 unrelated patients with the clinical suspicion of DMD/BMD. Most frequent deletions were: exons $48-50 \times 7$ (6.4\%), exons $45 \times 5$ (4.5\%), exons $45-47 \times 5$ (4.5\%) and exons 45-50 × 5 (4.5\%).

\begin{tabular}{|c|c|c|c|c|c|c|c|}
\hline Family & Mutation & $\begin{array}{l}\text { Reading } \\
\text { frame }\end{array}$ & Phenotype & Family & Mutation & $\begin{array}{l}\text { Reading } \\
\text { frame }\end{array}$ & Phenotype \\
\hline 1298 I/C & Del 1-18 & In & DMD & $1474 \mathrm{I} / \mathrm{NC}$ & Del 45-50 & Out & DMD \\
\hline 1345 I/C & Del 2-16 & Out & DMD & 1515 I/C & Del 45-50 & Out & $\mathrm{DMD}^{\mathrm{a}}$ \\
\hline 1364 & Del 3-7 & Out & DMD & $1306 \mathrm{I} / \mathrm{C}$ & Del 45-52 & Out & DMD \\
\hline 1343 & Del 3-9 & In & BMD & 1386 & Del 45-52 & Out & $\mathrm{DMD}^{\mathrm{a}}$ \\
\hline $1354 \mathrm{I} / \mathrm{C}$ & Del 3-9 & In & BMD & 1411 & Del 45-52 & Out & DMD \\
\hline $1392 \mathrm{I} / \mathrm{NC}$ & Del 3-11 & Out & IMD & 1497 & Del 45-52 & Out & DMD \\
\hline $1430 \mathrm{I} / \mathrm{NC}$ & Del 3-12 & In & $\mathrm{D} / \mathrm{BMD}$ & $1413 \mathrm{I} / \mathrm{C}$ & Del 45-53 & In & BMD \\
\hline 1396 & Del 3-20 & In & BMD & 1483 & Del 45-53 & In & BMD \\
\hline 1503 & Del 3-30 & In & DMD & 1408 & Del 45-54 & Out & DMD \\
\hline $1390 \mathrm{I} / \mathrm{C}$ & Del 4-7 & Out & $\mathrm{DMD}^{\mathrm{a}}$ & 1473 & Del 45-54 & Out & DMD \\
\hline 1333 I/C & Del 4-16 & In & DMD & 1332 & Del 45-55 & In & BMD \\
\hline $1400 \mathrm{I} / \mathrm{NC}$ & Del 5-7 & Out & DMD & 1353 & Del 45-55 & In & BMD \\
\hline 1461 I/C & Del 5-7 & Out & BMD & 1344 & Del 46-47 & Out & DMD \\
\hline 1490 & Del 5-7 & Out & DMD & $1429 \mathrm{I} / \mathrm{NC}$ & Del 46-47 & Out & DMD \\
\hline 1465 & Del 5-9 & In & BMD & $1457 \mathrm{I} / \mathrm{NC}$ & Del 46-47 & Out & DMD \\
\hline $1404 \mathrm{I} / \mathrm{C}$ & Del 6 & Out & DMD & 1301 & Del 46-48 & Out & DMD \\
\hline 1393 & Del 7-34 & Out & DMD & 1253 & Del 46-50 & Out & DMD \\
\hline 1297 & Del 8-9 & Out & DMD & 1453 & Del 46-51 & Out & DMD \\
\hline $1438 \mathrm{I} / \mathrm{NC}$ & Del 8-10 & Out & DMD & 1398 I/C & Del 46-52 & Out & DMD \\
\hline 1395 I/C & Del 8-13 & Out & DMD & 1489 I/NC & Del 46-52 & Out & DMD \\
\hline 1403 I/C & Del 9-20 & In & BMD & 1439 I/NC & Del 46-55 & Out & DMD \\
\hline 1480 & Del 10-21 & Out & DMD & 1347 & Del 47-50 & Out & DMD \\
\hline 1315 & Del 12-13 & Out & DMD & $1314 \mathrm{I} / \mathrm{C}$ & Del 48 & In & BMD \\
\hline 1447 & Del 12-25 & Out & DMD & $1428 \mathrm{I} / \mathrm{C}$ & Del 48 & In & BMD \\
\hline 1326 & Del 12-43 & In & BMD & 1523 & Del 48 & In & BMD \\
\hline 1266 & Del 13-41 & In & BMD & 1366 & Del 48-49 & In & BMD \\
\hline $1471 \mathrm{I} / \mathrm{C}$ & Del 16-43 & Out & DMD & 1401 & Del 48-49 & In & BMD \\
\hline 1278 & Del 17-43 & Out & DMD & 1317 I/NC & Del 48-50 & Out & DMD \\
\hline 1436 I/C & Del 18-21 & Out & DMD & 1319 I/C & Del 48-50 & Out & DMD \\
\hline $1324 \mathrm{I} / \mathrm{C}$ & Del 19-48 & In & $\mathrm{D} / \mathrm{BMD}$ & $1328 \mathrm{I} / \mathrm{NC}$ & Del 48-50 & Out & DMD \\
\hline $1318 \mathrm{I} / \mathrm{NC}$ & Del 20 & Out & DMD & 1346 & Del 48-50 & Out & DMD \\
\hline 1339 I/NC & Del 41-43 & Out & DMD & $1464 \mathrm{I} / \mathrm{NC}$ & Del 48-50 & Out & $\mathrm{DMD}$ \\
\hline 1342 & Del 44 & Out & DMD & 1466 & Del 48-50 & Out & DMD \\
\hline 1418 & Del 44 & Out & DMD & 1491 & Del 48-50 & Out & DMD \\
\hline $1426 \mathrm{I} / \mathrm{NC}$ & Del 44 & Out & DMD & 1275 & Del 48-51 & In & BMD \\
\hline 1376 & Del 44-47 & Out & $\mathrm{D} / \mathrm{BMD}$ & 1510 & Del 48-51 & In & BMD \\
\hline 1352 & Del 44-55 & Out & DMD & $1450 \mathrm{I} / \mathrm{C}$ & Del 48-52 & Out & DMD \\
\hline $1302 \mathrm{I} / \mathrm{NC}$ & Del 45 & Out & DMD & 1475 & Del 48-52 & Out & DMD \\
\hline 1355 & Del 45 & Out & DMD & $1280 \mathrm{I} / \mathrm{C}$ & Del 48-54 & Out & DMD \\
\hline 1368 & Del 45 & Out & DMD & 1460 I/NC & Del 48-56 & Out & DMD \\
\hline 1373 I/C & Del 45 & Out & DMD & 1300 & Del 49-50 & Out & DMD \\
\hline $1525 \mathrm{I} / \mathrm{NC}$ & Del 45 & Out & DMD & 1369 & Del 49-50 & Out & DMD \\
\hline 1287 & Del 45-47 & In & $\mathrm{BMD}$ & 1380 & Del 49-50 & Out & DMD \\
\hline $1321 \mathrm{I} / \mathrm{NC}$ & Del 45-47 & In & BMD & 1476 & Del 49-52 & Out & DMD \\
\hline 1350 & Del 45-47 & In & $\mathrm{D} / \mathrm{BMD}$ & $1495 \mathrm{I} / \mathrm{NC}$ & Del 49-54 & Out & DMD \\
\hline 1399 I/C & Del 45-47 & In & $\mathrm{D} / \mathrm{BMD}$ & 1335 & Del 50 & Out & BMD \\
\hline 1493 I/C & Del 45-47 & In & BMD & 1263 & Del 50-51 & In & BMD \\
\hline $1270 \mathrm{I} / \mathrm{NC}$ & Del 45-48 & In & BMD & 1414 I/NC & Del 51-55 & Out & DMD \\
\hline 1336 & Del 45-48 & In & BMD & $1256 \mathrm{I} / \mathrm{NC}$ & Del 52 & Out & DMD \\
\hline 1446 I/C & Del $45-48$ & In & BMD & 1292 & Del 52 & Out & DMD \\
\hline 1281 & Del 45-49 & In & BMD & $1513 \mathrm{I} / \mathrm{C}$ & Del 52 & Out & DMD \\
\hline 1459 I/NC & Del 45-49 & In & BMD & 1265 & Del 52-54 & Out & DMD \\
\hline 1377 I/NC & Del 45-50 & Out & DMD & 1517 & Del 53-54 & Out & DMD \\
\hline 1379 I/NC & Del 45-50 & Out & DMD & 1274 & Del 56-79 & Out & DMD \\
\hline 1449 I/C & Del 45-50 & Out & DMD & $1309 \mathrm{I} / \mathrm{C}$ & Del 65-79 & Out & DMD \\
\hline
\end{tabular}

$\mathrm{D} / \mathrm{BMD}$ - young patient, without possibility of observation and classification of clinical symptoms. I/C - isolated case of DMD/BMD, mother is a carrier. I/NC - isolated case of DMD/BMD, mother is not a carrier.

a Cases of mutations detected in women from the families with DMD examined in situation of lack of proband's DNA. 
Table 2 - Duplications in DMD gene detected by use of MLPA in the group of 180 unrelated patients with the clinical suspicion of DMD/BMD.

\begin{tabular}{|c|c|c|c|}
\hline Family & Mutation & $\begin{array}{c}\text { Reading } \\
\text { frame }\end{array}$ & Phenotype \\
\hline 1279 & Dup 2 & Out & DMD \\
\hline 1299 I/C & Dup $2-7^{b}$ & In & $\mathrm{DMD}^{\mathrm{a}}$ \\
\hline 1420 & Dup 3 & In & BMD \\
\hline 1506 & Dup 3-5 & In & DMD \\
\hline 1311 & Dup 4-7 & Out & DMD \\
\hline 1507 & Dup 5-18 & In & BMD \\
\hline $1422 \mathrm{I} / \mathrm{C}$ & Dup 7-15 & Out & DMD \\
\hline 1273 I/NC & Dup 8-11 & Out & DMD \\
\hline $1434 \mathrm{I} / \mathrm{C}$ & Dup 8-17 & Out & DMD \\
\hline 1486 & Dup 8-44 & Out & DMD \\
\hline 1295 & Dup 11-44 & In & BMD \\
\hline 1378 & Dup 12-13 & Out & DMD \\
\hline 1329 & Dup 28-43 & Out & DMD \\
\hline $1250 \mathrm{I} / \mathrm{C}$ & Dup 44 & Out & DMD \\
\hline 1500 & Dup 45-51 & In & DMD \\
\hline $1524 \mathrm{I} / \mathrm{C}$ & Dup 50 & Out & DMD \\
\hline 1299 & Dup 50-59 & In & $\mathrm{DMD}^{\mathrm{a}}$ \\
\hline 1271 & Dup 50-60 & In & DMD \\
\hline $1522 \mathrm{I} / \mathrm{C}$ & Dup 51-72 & Out & DMD \\
\hline 1249 & Dup 53 & Out & $\mathrm{D} / \mathrm{BMD}$ \\
\hline 1257 & Dup 63 & Out & DMD \\
\hline $1501 \mathrm{I} / \mathrm{NC}$ & Dup 64-67 & Out & DMD \\
\hline
\end{tabular}

D/BMD - young patient, without possibility of observation and classification of clinical symptoms. I/C - isolated case of DMD/ $\mathrm{BMD}$, mother is a carrier. $\mathrm{I} / \mathrm{NC}$ - isolated case of $\mathrm{DMD} / \mathrm{BMD}$, mother is not a carrier.

a Cases of mutations detected in women from the families with DMD examined in situation of lack of proband's DNA.

b Two independent duplications detected in one allele.

Small deletions involving less than 10 exons accounted for $79.1 \%(87 / 110)$ of all the deletions identified in the study, of which 3-exons deletions were the most frequent (19/110, $17.3 \%)$, followed by single-exon $(17 / 110,15.5 \%)$ and two-exon deletions (12/110, 10.9\%).

Exons $45-54(74 / 110,67 \%)$ and 3-21 (31/110, 28\%) were the most commonly deleted. Single-exon deletions occurred mainly in exons 44, 45, 48 and 52 (14/17). Exon 48 was the most frequently deleted (51/110, 46\%), followed by exon 49 (45/110, 41\%).

Small duplications involving less than 10 exons accounted for $63 \%(14 / 22)$ of all the duplications detected in this study, of which single-exon duplications were the most frequent $(6 / 22$, $27 \%)$. Exons 8-15 (7/22, 31\%) were most frequently duplicated. Exons 11-13 were most commonly duplicated (6/22, 27\%).

Frame-shift deletions were identified in $96 \%$ of DMD deletions (72/75). In frame deletions resulted in 93\% of BMD patients (27/29). Out of frame duplications were detected in $72 \%$ of DMD duplications (13/18); all three BMD duplications were in frame. Monaco's theory has been proved in $95 \%$ of deletions (99/ $104)$ and in $76 \%$ of duplications (16/21) in our study.

MLPA analysis showed presence of a mutation in 34 of 62 mothers from DMD/BMD families with a single affected male (56\%). Deletion was identified in 28 females (28/54 52\%) and duplication - in 6 (6/8 75\%) (Tables 1 and 2). In 4 of these carriers mutations were identified in absence of DNA from probands (3 deletions, 1 tandem duplication). Cases representing "de novo" mutation, deletion or duplication, account for $44 \%(28 / 62)$.

\section{Discussion}

Multiple ligation-dependent probe amplification (MLPA) is a very reliable method for detection deletions and duplications in DMD/BMD patients $[21-23,26]$. Deletions have been identified in $61 \%$ of patients, duplications - in $12 \%$, point mutations in two cases $(1 \%)$, which resulted in sensitivity of mutation detection by MLPA of $74 \%$. Our data confirm an observation that deletions are more frequently found in BMD patients (78\%) than DMD patients (56\%). MLPA analysis showed presence of a deletion or duplication in $86 \%$ of BMD patients and in $69 \%$ DMD patients. A search for point mutations in the remaining patients (26\%) is going to be carried following immunohistochemistry of the muscle samples to confirm clinical diagnosis. Point mutations occurring in sequences hybridizing to the MLPA probes may be detected as false-negative results $[21,27]$. Two point mutations identified in this study, were first identified as MLPA single exon deletions. Both exons, were successfully amplified by standard PCR and sequenced, showing presence of point mutations: c.134delA in exon 3 (p.Q45Rfs*7) and c.5407C>T in exon 38 (p. Q1803*).

Location of deletions and duplications, detected in this study, stayed in agreement with data published by other authors [21-23,26-28]. Deletions clustered within two hot spots, mayor encompassing exons 44-52 (68\%) and minor site including exons 3-19 (28\%). The highest concentration of duplications was found in proximal part of the gene, between exons 8 and 15 (27\%) (Fig. 1). Distribution of breakpoints (Fig. 2) is in accordance with the published data for the West-European population [29].

Like in our previous report [28], small deletions, including less than 10 exons, were found to be predominant in this study (84\%). We also confirm an observation made by other researchers that majority of duplications are small ones, encompassing less than 10 exons (73\%) [10,13-15].

Off all the exons, exon 48 was the most frequently deleted, followed by exon 49, and exons 50; exons 11-13 were most commonly duplicated.

According to Monaco's theory, clinical severity of the disease results from either disruption or preservation of the reading frame by a mutation. Frameshifting mutations usually cause DMD, while in BMD the reading frame stays intact. Monaco's theory complies with phenotype in 90\% [13-15]. In our study $96 \%$ of deletions in DMD and 93\% of deletions in BMD were consisted with the reading frame rule. In our study, two cases of BMD resulted from out of frame deletions (exons 5-7 and 50), inconsistently with Monaco's rule. Most likely additional exons were removed by alternative splicing, restoring reading frame: exon 8 in the first and exon 51 in the second case. Out of frame deletion of exons 5-7 was also detected in two DMD patients. Different phenotypes resulting from the same mutation were also reported by other researches [30]. Accuracy of the model for duplications is lower and should be applied carefully [14]. Out of frame duplications were found in $72 \%$ of DMD patients. This is in accordance with the results of other authors $[14,19,30]$. Less regular accordance with the reading frame rule in cases of 


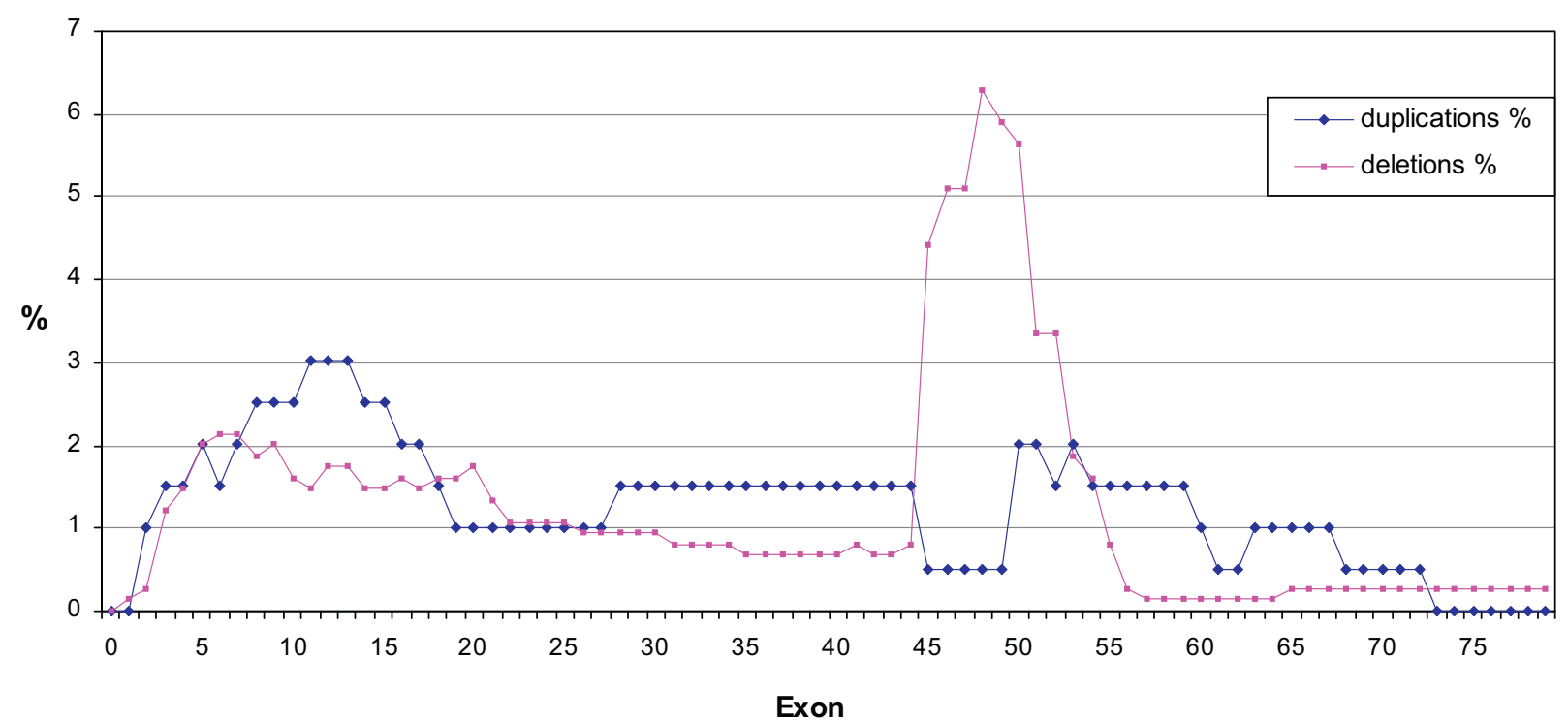

Fig. 1 - Relative frequency of deletions and duplications in exons of dystrophin gene for 110 deletions and 22 duplications detected by use of MLPA in the group of 180 unrelated patients with the clinical suspicion of DMD/BMD.

duplications may be explained by their effects on splicing RNA and the resulting synthesis of dystrophin. The inverted duplications were reported [20] to cause disruption the transcription [26].
Identification of female carriers is essential for DMD/BMD families, since there is no effective treatment for the disease. In the families with a single affected male, it is fundamental to determine whether the disease resulted from a 'de novo'
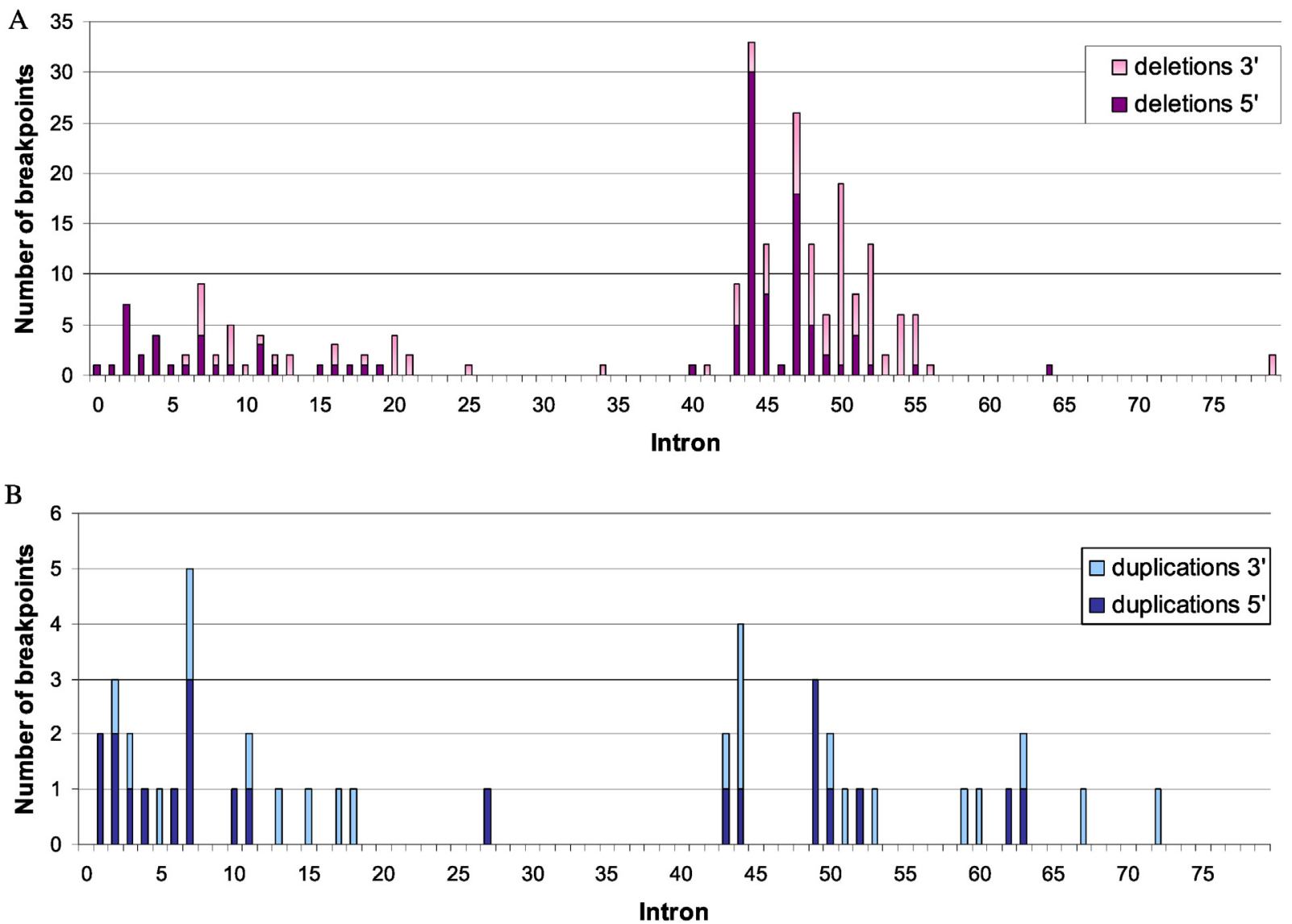

Fig. 2 - Distribution of intronic breakpoints of deletions (A) and duplications (B) within the dystrophin gene. The number of starting breakpoints ( $5^{\prime}$ breakpoint, black) and ending breakpoints ( $3^{\prime}$ breakpoint, grey bars) of either deletions and duplications respectively localized in each intron are indicated. 
mutation or a genetic defect, inherited from a carrier mother. MLPA technique is a very useful tool for mutation detection in female carriers, in which deletions and duplications are not detectable in by standard PCR method [21-23,27]. In our study $56 \%$ of the mothers of single affected males were found to be carriers of mutations, in remaining $44 \%$ carriership was ruled out proving the occurrence of 'de novo' mutations. Similar results were published for the populations of North India [31], Mexico [32] and Australia [33]. French results, however, were different; de novo mutations were reported only in $24.5 \%$ of the isolated cases [13]. The differences may be due to statistical biases e.g. - probability of familial cases being recorded is higher than in isolated cases; it is also possible that some noncarrier females - mothers of DMD/BMD isolated cases in our material are in fact germinal mosaics.

MLPA is also a powerful tool for carriership analysis in DMD families, in which DNA sample from patient is not available. In our study four of the mothers were found to be carriers of mutations detected in absence of DNA sample from a patient (three deletions, one case of two independent duplications).

MLPA technique seems to be a reliable tool in the screening of deletions and duplications in the dystrophin gene both in DMD/ BMD patients and potential female carriers, especially those, who cannot be investigated by other methods. The mutation data resulted from this study may be used for and experimental gene therapy of DMD patients, involving "exon skipping" approach [34] and further genotype/phenotype studies. The results of carriership status analysis are an essential tool for genetic counseling and prenatal diagnosis in DMD/BMD families.

\section{Conclusions}

1. Localization of "hot spots" in the dystrophin gene is different for deletions and duplications.

2. Precise determination of size and location of a deletion in the dystrophin gene allows for prediction of clinical form of the dystrophy; in case of duplication, this correlation is lower.

3. MLPA technique is a quick, convenient and efficient tool for deletion and duplication detection both in affected patients and female carriers, therefore carriership testing of DMD/ BMD is available in absence of DNA sample from an affected person.

\section{Conflict of interest}

None declared.

\section{Acknowledgement and financial support}

The authors would like to thank medical doctors from many diagnostic centers in the country for directing DMD/BMD patients for molecular diagnosis in the Department of Genetics of the Institute of Psychiatry and Neurology.

The work was partly supported by Grant nr 641/N-TREAT/ 09/2010/0 from the Ministry of Science and Higher Education.

\section{Ethics}

The work described in this article has been carried out in accordance with The Code of Ethics of the World Medical Association (Declaration of Helsinki) for experiments involving humans; Uniform Requirements for manuscripts submitted to Biomedical journals.

\section{R E F E R E N C E S}

[1] Emery AE. Population frequencies of inherited neuromuscular diseases: a world survey. Neuromuscul Disord 1991;1:19-29.

[2] Helderman-van den Enden A, Ginjaar H, Bakker E. Impact of prenatal diagnosis on the incidence of DMD in the Netherlands. Neuromuscul Disord 2007;17:764-900.

[3] Passamano L, Taglia A, Palladino A, Viggiano E, D'Ambrosio P, Scutifero M, et al. Improvement of survival in Duchenne muscular dystrophy: retrospective analysis of 835 patients. Acta Myol 2012;31(October (2)):121-5.

[4] www.treat-nmd.eu.

[5] Jefferies JL, Eidem BW, Belmont JW, Craigen WJ, Ware SM, Fernbach SD, et al. Genetic predictors and remodeling of dilated cardiomyopathy in muscular dystrophy. Circulation 2005;112:2799-804.

[6] Koenig M, Hoffman EP, Bertelson CJ, Monaco AP, Feener C, Kunkel LM. Complete cloning of the Duchenne muscular dystrophy (DMD) cDNA and preliminary genomic organization of the DMD gene in normal and affected individuals. Cell 1987;50(July (3)):509-17.

[7] Muntoni F, Torelli S, Ferlini A. Dystrophin and mutations: one gene, several proteins, multiple phenotypes. Lancet Neurol 2003;2:731-40.

[8] Ervasti JM, Campbell KP. Membrane organization of the dystrophin-glycoprotein complex. Cell 1991;66: 1121-31.

[9] Hoffman EP, Kunkel LM, Angelini C, Clarke A, Johnson M, Harris JB. Improved diagnosis of Becker muscular dystrophy by dystrophin testing. Neurology 1989;39:1011-7.

[10] Den Dunnen JT, Grootscholten PM, Bakker E, Blonden LA, Ginjaar HB, Wapenaar MC, et al. Topography of the Duchenne muscular dystrophy (DMD) gene: FIGE and cDNA analysis of 194 cases reveals 115 deletions and 13 duplications. Am J Hum Genet 1989;45:835-47.

[11] Forrest SM, Cross GS, Flint T, Speer A, Robson KJH, Davies KA. Further studies of gene deletions that cause Duchenne and Becker muscular dystrophies. Genomics 1988;2 (February (2)):109-14.

[12] Hu XY, Ray PN, Murphy EG, Thompson MW, Worton RG. Duplicational mutation at the Duchenne muscular dystrophy locus: its frequency, distribution, origin, and phenotype-genotype correlation. Am J Hum Genet 1990;46:682-95.

[13] Tuffery-Giraud S, Beroud C, Leturcq F, Yaou RB, Hamroun D, Michel-Calemard L, et al. Genotype-phenotype analysis in 2,405 patients with a dystrophinopathy using the UMDDMD database: a model of nationwide knowledgebase. Hum Mutat 2009;30:934-45.

[14] Takeshima Y, Yagi M, Okizuka Y, Awano H, Zhang Z, Yamauchi $Y$, et al. Mutation spectrum of the dystrophin gene in 442 Duchenne/Becker muscular dystrophy cases from one Japanese referral center. J Hum Genet 2010;55:379-88. 
[15] Yang J, Li SY, Li YQ Cao JQ Feng SW, Wang YY, et al. MLPA-based genotype-phenotype analysis in 1053 Chinese patients with DMD/BMD. BMC Med Genet 2013;14:29.

[16] Roberts RG, Gardner RJ, Bobrow M. Searching for the 1 in 2,400,000: a revive of dystrophin gene point mutations. Hum Mutat 1994;4(1):1-11.

[17] Monaco AP, Bertelson CJ, Liechti-Gallati S, Moser H, Kunkel LM. An explanation for the phenotypic differences between patients bearing partial deletions of the DMD locus. Genomics 1988;2:90-5.

[18] Koenig M, Beggs AH, Moyer M, Scherpf S, Heindrich K, Bettecken T, et al. The molecular basis for Duchenne versus Becker muscular dystrophy: correlation of severity with type of deletion. Am J Hum Genet 1989;45:498-506.

[19] Gualandi F, Neri M, Bovolenta M, Martoni E, Rimessi P, Fini $\mathrm{S}$, et al. Transcriptional behavior of DMD gene duplications in DMD/BMD males. Hum Mutat 2009;30(2):E310-9.

[20] Ishmukhametova A, Chen JM, Bernard R, de Massy B, Baudat F, Boyer A, et al. Dissecting the structure and mechanism of a complex duplication-triplication rearrangement in the DMD gene. Hum Mutat 2013;34 (August (8)):1080-4.

[21] Janssen B, Hartmann C, Scholz V, Jauch A, Zschocke J. MLPA analysis for the detection of deletions, duplications and complex rearrangements in the dystrophin gene: potential and pitfalls. Neurogenetics 2005;6:29-35.

[22] Gatta V, Scarciolla O, Gaspari AR, Palka C, de Angelis MV, Di Muzio A, et al. Identification of deletions and duplications of the DMD gene in affected males and carrier females by multiple ligation probe amplification (MLPA). Hum Genet 2005;117:92-8.

[23] Lai KKS, Lo IFM, Tong TMF, Cheng LYL, Lam STS. Detecting exon deletions and duplications of DMD gene using multiplex ligation-dependent probe amplification (MLPA). Clin Biochem 2006;39:367-72.

[24] Bennett RR, den Dunnen J, O'Brien KF, Darras BT, Kunkel LM. Detection of mutations in the dystrophin gene via automated DHPLC screening and direct sequencing. Genetics 2001;2:17.
[25] Zimowski J, Fidziańska E, Holding M, Zaremba J. Two mutations in one dystrophin gene. Neurol Neurochir Pol 2013;47(2):131-7.

[26] White SJ, Aartsma-Rus A, Flanigan KM, Weiss RB, Kneppers AL, Lalic T.. et al. Duplications in the DMD gene. Hum Mutat 2006;27:938-45.

[27] Schwartz M, Duno M. Improved molecular diagnosis of dystrophin gene mutation using the multiplex ligationdependent probe amplification method. Genet Test 2004;8:361-7

[28] Zimowski JG, Bisko MU, Fidziańska EJ, Fidzianska AZ, Badurska B, Nieboj-Dobosz I, et al. Detection of deletions within the dystrophin gene in Polish families affected with Duchenne/Becker muscular dystrophy. Eur J Neurol 1997;4:138-42.

[29] Danieli GA, Mioni F, Muller CR, Vitiello L, Mostacciuolo ML, Grimm T, et al. Pattern of deletions of the dystrophin gene in different European populations. Hum Genet 1993;91:342-6.

[30] Kesari A, Pirra LN, Bremadesam L, Marcintyre O, Gordon E, Dubrovsky AL, et al. Integrated DNA, CDNA, and proteins studies in Becker muscular dystrophy show high exception to the reading frame rule. Hum Mutat 2008;29:728-37.

[31] Sinha S, Mishra S, Singh V, Mittal RD, Mittal B. High frequency of new mutations in North Indian Duchenne/ Becker muscular dystrophy patients. Clin Genet 1996;50 (5):327-31.

[32] Alcántara MA, Villarreal MT, Del Castillo V, Gutirrez G, Saldana Y, Maulen I, et al. High frequency of de novo deletions in Mexican Duchenne and Becker muscular dystrophy patients. Implications for genetic counseling. Clin Genet 1999;55(5):376-80.

[33] Taylor PJ, Maroulis S, Mullan GL, Pedersen RL, Baumli A, Elakis G, et al. Measurement of the clinical utility of a combined mutation detection in carriers of Duchenne and Becker muscular dystrophy. J Med Genet 2007;44:368-72.

[34] Aartsma-Rus A, van Ommen GJB. Antisense-mediated exon skipping: a versatile tool with therapeutic and research applications. RNA 2007;13:1609-24. 